\title{
Téoros
}

Revue de recherche en tourisme

\section{Les parcs : des réseaux en mutation}

\section{Roger Nadeau}

Volume 14, numéro 1, printemps 1995

Les parcs : des réseaux en mutation

URI : https://id.erudit.org/iderudit/1077046ar

DOI : https://doi.org/10.7202/1077046ar

Aller au sommaire du numéro

Éditeur(s)

Université du Québec à Montréal

ISSN

0712-8657 (imprimé)

1923-2705 (numérique)

Découvrir la revue

Citer ce document

Nadeau, R. (1995). Les parcs : des réseaux en mutation. Téoros, 14(1), 2-2.

https://doi.org/10.7202/1077046ar d'utilisation que vous pouvez consulter en ligne.

https://apropos.erudit.org/fr/usagers/politique-dutilisation/ 
Ce n'est pas par hasard si la revue Téoros a choisi de présenter un numéro thématique sur les parcs à ce momentci. L'année 1995 coincide en effet avec le centième anniversaire de la création du premier parc au Québec. celui de la Montagne Tremblante dans les Laurentides montréalaises, ainsiqu'avecle vingt-cinquième anniversaire de l'implantation de parcs nationaux en sol québécois (les parcs de Forillon et de la Mauriciel. II nous semble que le momentest donc particulièrement bien choisipour favoriser une réflexion élargie sur ce segment de notre patrimoine naturel. On peutd'ailleurs observer que les contextes géopolitiques et socio-économiques se prêtent fort bien à ces réflexions alors qu'au Québec et au Canada des pratiques courantes sont questionnées ou remises en question.

Ce premier numéro de Téoros sur les parcs naturels n'a pas la prétention de vouloir répondre a toutes les problématiques qui peuvent être posées mais peut-être davantage d'amorcer une réflexion ou une analyse porteuse d'avenir. D'ores et déjà, une constatation s'impose: en dehors des gestionnaires de parcs associés à la fonction publique fédérale ou québécoise, très peu de recherches, de réflexions ou d'analyses approfondies sont réalisées au Québec sur le réseau québécois en comparaison avec ce que l'on trouve dans la plupart des autres provinces canadiennes, aux États-Unis et dans la plupart des pays d'Europe de l'Ouest.

S'agit-il d'un désintéressement des chercheurs québécois vis-à-vis leur réseau de parcs?

En lisant les articles qui nous sont parvenus dans le cadre de ce numéro sur les parcs, on peut noter que certaines préoccupations rejoignent plusieurs auteurs. On pense ici particulièrement à la place de lhomme dans le milieu naturel d'une part et d'autre part, on cherche la zone d'équilibre entre lamission de protection de l'environnement et la mise en disponibilite de ces espaces a des fins touristiques ou de récréation pour une population qui ressent de plus en plus le besoin de se retrouver dans une nature intacte et bien vivante.

Il fut un temps encore tout proche où créer un parc impliquait des procédures d'expropriation qui visaient à expulser parla force les gens quihabitaient ce territoire que soudain l'État avait commencé à convoiter pour en faire un parc. Manifestement, on prenait donc pour acquis que I'homme ne faisait pas parti du milieu naturel et que la présence de ce wprédateur n devait à tout prixêtre éliminée. Assezétrangement, quandle parc était ouvertaupublic, on s'empressait d'offrir à la clientèle touristique des activités d'interprétation qui visaient à reconstituer les modes de vie qui se pratiquaient sur ce territoire dans le passé. Par exemple, on pouvait faire disparaître de typiques petits villages de pêcheurs pour, quelques annees plus tard, engager pour la période estivale des étudiants que l'on va déguiser en pêcheurs et qui vont expliquer aux visiteurs ce qu'était la "vraie vien des gens qui vivaient ici avant... II semble bien que cette étrange logique ait fait son temps car aujourd'huiau contraire, on chercheả intégrerl'homme dans son milieu naturel quotidien et réel car dans les faits, qui est le mieux placé pour parler de la vie des villages de pêcheurs que le pêcheur lui-même qui peut agrémenter son propos d'une foule d'anecdotes vécues. Cettenouvelle approche est maintenant admise et acceptée par à peu près tous les intervenants, de sorte que lon peut croire que l'époque des expropriations sauvages est terminée et que désormais l'homme est appréhendé comme étant une composante de ce milieu naturel qui suscite tant d'intérêt.

Un autre volet de reflexion qui revient souvent concerne en fait la mission spécifique des parcs. Bien sûr, on a développé des parcs de conservation et des parcs de récréation dans le réseau québécois et cette appellation témoigne de missions diverses. Mais quelle que soit la catégorie de parc, il y aura toujours une préoccupation majeure orientée vers la protection de l'environnementen méme temps qu'une préoccupation toute aussi majeureả mettre ces espaces a la disposition des vacanciers. A priori, on peut se demander si ces deux objectifs sont vraiment conciliables. Est-ce que l'augmentation des flux touristiques en faveur des parcs met en péril leur intégrité écologique? La plupart du temps, une troisième variable, celle de la rentabilité, vient compliquer cette équation, surtout aujourd'hui où nos gouvernements s'empêtrent dans des déficits qui semblent devenus incontrólables. Est-ce que la recherche de la rentabilité est susceptible de compromettre l'accessibilité pour tous par l'imposition d'une nouvelle tarification généralisée?

Comme on peut le constater, bien des questions peuvent se poser relativement à la mission, à la gestion et à la fréquentation des parcs. Comme je le signalais au début, ce numéro de Téoros ne va pas répondre à tout mais il voudrait au moins vous mettre l'eau à la bouche. Nous avons donc regroupé ici une brochette de spécialistes de la question et chacun transmet une partie de son expertise, sur des parcs québécois, sur des parcs nationaux et méme sur des parcs européens. II nous apparait que toutes les personnes préoccupées par le devenir de ces espaces naturels exceptionnels tireront grand profit de l'expérience vécue parl'ensemble de ces acteurs que nous remercions chaleureusement pour leur précieuse collaboration

Bonne lecture !

Roger Nadeau,

Coordonnateur 